Published in final edited form as:

Open Conf Proc J. 2010 ; 1: 1-6. doi:10.2174/2210289201001010001.

\title{
MORPHEEINS - A NEW PATHWAY FOR ALLOSTERIC DRUG DISCOVERY
}

\author{
EILEEN K. JAFFE \\ Institute for Cancer Research, Fox Chase Cancer Center, 333 Cottman Ave, Philadelphia, PA \\ 19111 U.S.A
}

\section{Abstract}

The morpheein model of allosteric regulation can be applied as a novel approach to the discovery of small molecule allosteric modulators of protein function. Morpheeins are homo-oligomeric proteins where, under physiological conditions, the oligomer can dissociate, the dissociated units can change conformation, and the altered conformational state can reassociate to a structurally and functionally distinct oligomer. This phenomenon serves as a basis for allostery, as a basis for conformational diseases, as a basis for drug discovery, and may be applicable to personalized medicine such as in the prediction of drug side effects. Each of these relationships has been established for the prototype morpheein, porphobilinogen synthase, where the conformational disease is a porphyria and the drug application is in antimicrobial discovery. These data are presented along with a discussion of other drug targets for which the morpheein model of allostery may apply. Such targets include HIV integrase, TNF $\alpha, \beta$-tryptase, and p53.

\section{INTRODUCTION}

Proteins carry out the bulk of the physical and catalytic work of the cell. Errors in protein function provide a basis for disease and, consequently, control of protein function is a goal in drug development. Although early work in protein crystallography encouraged a view of protein structure as largely static, the $21^{\text {st }}$ century view emphasizes the flexibility of proteins and how protein structure dynamics are essential to protein function. This article focuses on a newly discovered mode of protein quaternary structure dynamics that promises both to deepen and broaden our understanding of protein structure-function relationships and our ability to control protein function.

Allostery provides a mechanism for functional control of proteins, specifically enzymes, which bypasses the inherent limitations of active site targeting [1]. Allosteric regulators control enzyme function through binding to a site other than the active site; allosteric sites are generally less conserved than active sites. Thus, allostery provides a potential mechanism for species-selective inhibition of an essential, but highly conserved enzyme, as might be useful for the development of new antimicrobials. Allosteric regulators also provide for modulation of a specific enzyme among a large class of functionally similar enzymes (e.g. kinases or phosphatases). Hence, therapeutics that function as allosteric regulators are attractive targets for development [2]. This article focuses on morpheeins and the morpheein model of allosteric regulation as a novel basis for drug discovery.

\section{Morpheeins and the Morpheein model of Allostery Regulation}

Morpheeins are homo-oligomeric proteins that can dissociate, change conformation in the dissociated state, and then reassemble to an alternate oligomer [3]. In the dissociated state, one conformation dictates assembly to a particular oligomer and the alternate conformation dictates assembly to an alternate oligomer. A dice model, Fig (1a), is useful for illustrating 
how a morpheein accomplishes its quaternary structure rearrangement. Unlike proteins that can form amyloid (e.g. prions), the conformational change between morpheein forms (components of the equilibrium) does not involve changes in the core protein fold. Rather, the conformational change is characterized by a small number of adjustments in backbone and side chain angles that are kinetically feasible under physiologic conditions. Also, unlike amyloid forming proteins, the alternate oligomer stoichiometries are finite.

The alternate quaternary structure assemblies of a morpheein equilibrium have alternate functions, each of which is physiologically relevant [3]. Examples of such alternate functions include, but are not limited to: 1) "on" vs. "off" states, 2) states having different interaction partners in a signaling pathway, 3) states having different affinity or specificity for allosteric effector molecules, and 4) states having different "moonlighting" functions. The ability of proteins to participate in such a reversible quaternary structure dynamic provides one way that a limited number of encoded genes can provide a much larger number of functions. Most significantly, the transition between alternate assemblies provides a mode of allostery. The morpheein model of allosteric regulation is illustrated in Fig (1b). The obligate oligomer dissociation step distinguishes this model of allostery from the more traditional concerted Monod-Wyman-Changeux and sequential Koshland-Nementhy-Filmer models $[4,5]$, although the morpheein model is also concerted.

\section{PORPHOBILINOGEN SYNTHASE}

The quaternary structure dynamic illustrated in Fig (1) became known to us through our work with porphobilinogen synthase (PBGS), which is an enzyme that catalyzes the first common step in the tetrapyrrole biosynthetic pathway (e.g. heme, chlorophyll, vitamin $\mathrm{B}_{12}$ ). Our extensive and innovative characterization of the PBGS family of enzymes has taught us that the assembly state is of the utmost importance to PBGS activity and that there are significant phylogenetic differences between the amino acids that govern the equilibrium of PBGS assemblies [6-11]. Although the active site is not at a subunit interface, there are intersubunit interactions that contribute to the structure and dynamics of an active site lid and thus effect catalytic activity [10]. In the case of PBGS, the kinetics and thermodynamics of the quaternary structure equilibria are phylogenetically variable because many of the amino acids that make up the subunit interfaces are not conserved. For PBGS of different species, the quaternary structure equilibrium serves different functions and can be exploited for different purposes. It is a basis for allostery, a basis for a conformational disease, a basis for drug discovery, and may be applicable to personalized medicine in the prediction of drug side effects. The PBGS quaternary structure equilibrium is described below, and the varied aspects of PBGS structure and function are discussed.

\section{The Structure of PBGS}

PBGS exists in a dynamic equilibrium of high activity octamers and low activity hexamers, and the interconversion of these two oligomers involves a conformational change at the level of the dimer. This quaternary structure dynamic is illustrated in Fig (2), where the stoichiometry is different from Fig (1a), but the mechanism is the same [3,8,9,11-17]. Oligomers with subtle sequence differences and the technique of mass spectrometry have been used to definitively establish the dissociative mechanism in the hexamer to octamer conversion process [17]. Native polyacrylamide gel electrophoresis, dynamic light scattering, and kinetic hysteresis have been used to monitor the quaternary structure interconversion process $[9,11,12,16-18]$. In order for this equilibrium to be physiologically relevant, it is important that hexamer and/or octamer dissociation yield dimers where the newly solvent-exposed surfaces are largely hydrophilic. For PBGS there are two possible mechanisms for oligomer dissociation (Fig (2)) and based on the hydrophilicity of the 
exposed surfaces, it appears that PBGS from different species may use different dissociation mechanism.

\section{Allosteric Regulation in PBGS}

The allosteric regulation of PBGS by magnesium is physiologically relevant in plants. X-ray crystallography has shown how a naturally occurring allosteric activator, magnesium, stabilizes the octamer, thus increasing activity [19]. PBGS from photosynthetic eukaryotes contains an allosteric magnesium binding site at a subunit interface that is specific for the high activity octamer. The octamer-specific location of the allosteric magnesium binding site is included in Fig (2). At low magnesium, plant PBGS, which is located in the chloroplast, exists as the low activity hexamer. Because the intermediates in chlorophyll biosynthesis are highly photo-reactive, it is essential that chlorophyll biosynthesis be tightly controlled such that there is no accumulation of these toxic intermediates. It is well established that one of the first events that occurs when a greening plant is exposed to light is the flooding of the chloroplast with magnesium [20]. The pre-flooding concentration of magnesium is below the $K_{\mathrm{d}}$ of the allosteric magnesium. The post-flooding concentration is saturating for this allosteric magnesium, such that the magnesium influx shifts the equilibrium toward the octamer and contributes to turning on chlorophyll biosynthesis. It is interesting to note that, in addition to plants, the allosteric magnesium binding site is also present in PBGS from nearly all bacteria, archaea, and many unicellular eukaryotes [21]. It is not present in the PBGS from metazoa or fungi. The evolutionary pressure that drove this segregation is not at all clear. Nearly all of those PBGS that do not contain the allosteric magnesium binding site contain instead an arginine residue whose guanidinium group is spatially equivalent to the allosteric magnesium. This arginine also helps stabilize the octamer [16].

Although human PBGS does not contain the allosteric magnesium binding site, it too can be regulated allosterically through oligomer-specific ligand binding. In this case the regulation is caused by small molecule binding to the hexameric assembly, which inactivates the protein. This phenomenon is decribed below in the context of the now well characterized quaternary structure equilibrium of human PBGS.

\section{Human PBGS Quaternary Structure Equilibrium}

Under normal physiologic conditions, wild type human PBGS populates the octameric and hexameric assemblies, with a very small fraction present as the dimer [12]. At basic $\mathrm{pH}$ the equilibrium of quaternary structure assemblies is drawn toward the hexamer and this phenomenon is responsible for a $\mathrm{pH}$ dependent loss in activity. We determined that the two common alleles for human PBGS have a slightly different propensity to populate the hexameric assembly and asked whether disease associated alleles encode protein with a greater propensity to form hexamer.

The Conformational Disease ALAD-Porphyria-ALAD-porphyria is a rare genetic disease whose patients are compound heterozygotes for two of eight known genes that encode defective PBGS proteins [22]. Carriers, who have only one defective gene, have about 50\% the normal PBGS activity, but are generally healthy in the absence of secondary factors. Most of the ALAD-porphyria associated mutations are not at the enzyme active site. We found that all of the ALAD-porphyria associated mutations yield proteins that form hexamer more readily than either of the wild type alleles. Hence, one can consider ALADporphyria a conformational disease [15]. We have proposed that diseases caused by a shift in such a quaternary structure equilibrium can broaden the definition of conformational diseases beyond those involving amyloid or other misfolding events [23]. In the case of ALAD-porphyria, small molecules that stabilize the "healthy" quaternary structure assembly 
(octamer) may provide a therapeutic advantage. However, the exceedingly rare nature of ALAD-porphyria argues against PBGS as a viable target for the development of octamer stabilizing therapeutics. Nevertheless, oligomer stabilizing small molecules constitute a viable method to control the function (e.g. activate or inhibit) of proteins that function as morpheeins.

Off-Target Drug Side Effects and Personalized Medicine-We have considered whether there are small molecules in the human environment (foods, drugs, environmental contaminants) that can preferentially bind to and stabilize the human PBGS hexamer and inhibit enzyme activity. The hexameric assembly of PBGS contains an oligomer-specific surface cavity, illustrated in Fig (2), that is not present in the octamer. Small molecules that bind to this hexamer-specific surface cavity will stabilize the hexamer, draw the quaternary structure equilibrium toward the hexamer, and inhibit enzymatic activity. If such molecules derive from foods, drugs, or environmental contaminants, then these have the capacity to inhibit human PBGS activity and cause physiologic responses reminiscent of porphyria and/ or lead poisoning, for which PBGS inhibition is a primary effect. Using a computational docking and an in vitro validation approach, we have identified two amebicidal drugs that stabilize the human PBGS hexamer in vitro [8]. The side effects of these drugs are consistent with inhibition of human PBGS. Knowledge of which proteins are morpheeins, which drugs alter their quaternary structure equilibrium, and an understanding of how allelic differences effect the quaternary structure equilibrium has promise for application to personalized medicine.

\section{Allosteric Inhibition of PBGS as a Foundation for Antimicrobials and Herbicides}

Normally one would not consider the highly conserved and essential tetrapyrrole biosynthetic pathway for antimicrobial or herbicide development. However, we can exploit differences between the components of the quaternary structure equilibrium of PBGS from different organisms and phylogenetic differences in the hexamer-specific surface cavity. For instance, we now know that some PBGS (e.g. human, pea, Yersinia enterocolitica) participate in the formation of octamers, dimers, and hexamers, while others (e.g. Pseudomonas aeruginosa and Toxoplasma. gondii) populate only the octameric and dimeric states [7]. Our success in the discovery of species-selective allosteric PBGS inhibitors to date has focused on the hexamer-specific surface cavity $[6,8,9,24]$. This surface is phylogenetically variable and contains topologies (crevices) that can serve as small molecule binding sites. Such surface cavities provide opportunities for small molecule allosteric regulation of PBGS activity and a novel approach to PBGS inhibition that avoids targeting the highly conserved enzyme active site. Although we and others have investigated active site directed inhibition of PBGS, potent and species specific active site inhibitors of PBGS from a human pathogen have not been reported [19,25-31]. Species-selective inhibitors that function by stabilizing the hexamer of pea PBGS and compounds that stabilize the hexamer of $Y$. enterocolitica PBGS were discovered by computational docking of small molecule libraries from Life Chemicals, Inc., to the hexamer-specific surface cavity using the program GLIDE and other components of the Schrodinger software suite [32,33]. The mechanism of inhibition is established to be via hexamer stabilization and human PBGS is not inhibited [9,24]. Although we have not established that inhibition of PBGS will kill plants or $Y$. enterocolitica, it is established that PBGS inhibition in $T$. gondii can clear the parasite from infected fibroblasts [7], suggesting promise for targeting PBGS in the apicomplexan parasites that cause toxoplasmosis and malaria.

\section{Application of the Morpheein Behavior of PBGS to the Science of Therapeutics}

PBGS is the prototype morpheein and though it may only have limited application in the science of therapeutics, its behavior provides a road map for how one might approach other 
proteins that function as morpheeins. For instance, it is known that TNF $\alpha$ can be inhibited by a compound that stabilizes a dimer, while TNF $\alpha$ is known to exist primarily as a trimer [34]. It is known that HIV integrase participates in a quaternary structure equilibrium that can be modulated by peptides that have been named shiftides $[35,36]$. It is known that $\beta$ tryptase exists as functionally distinct tetramers that are slow to interconvert, consistent with a system that must dissociate, change conformation, and reassociate differently [37]. It is known that p53, which is mutated in more than half of all cancers, forms a tetramer when it interacts with DNA, but its core domain can assemble as a trimer in the absence of DNA [38]. This, taken with the knowledge that $\mathrm{p} 53$ has important cytosolic functions (where DNA is not present), suggests that some important p53 functions may be as a trimer. Although these proteins are not yet established to function as morpheeins, they, and other proteins, are reported to have behaviors that are consistent with the morpheein view of protein quaternary structure dynamics. The approach of stabilizing one or another of the quaternary structure assemblies that comprise the morpheein equilibrium is proposed to provide a new and exciting approach to understanding and controlling the function of proteins, which is, after all, a primary mode of action for small molecule therapeutics.

\section{SUMMARY}

Using PBGS as an example, we show that 1) naturally occurring mutations to may perturb the normal balance of quaternary structure assemblies (with alternate functions) and contribute to disease states; 2 ) stabilization of one oligomer in an equilibrium of quaternary structure assemblies may provide a general mechanism for off-target drug side effects; 3 ) the morpheein model of allostery provides a new structural framework for understanding the physiological control of protein function; and 4) that targeting an oligomer-specific surface cavity on a protein that exists in an equilibrium of non-additive quaternary structure assemblies provides a new approach to allosteric drug discovery. The morpheein view of protein quaternary structure dynamics is one of a growing number of exceptions to the onesequence-one-structure-one-function paradigm, which has dominated our view of protein structure and function. Consideration of a protein as a morpheein adds a quaternary structure component to protein structure dynamics in the understanding of disease and in targeting protein structure dynamics in the development of new therapeutics.

\section{Acknowledgments}

This work was supported by grants from the National Institutes of Health, ES003654 (EKJ), AI077577 (EKJ), CA006927 (Inst. for Cancer Research), and CA009835 (Fox Chase Cancer Center), and by generous support from the Fox Chase Cancer Center.

\section{ABBREVIATIONS}

PBGS porphobilinogen synthase

\section{References}

1. Monod J, Changeux JP, Jacob F. Allosteric proteins and cellular control systems. J Mol Biol. 1963; 6:306-29. [PubMed: 13936070]

2. Hardy JA, Wells JA. Searching for new allosteric sites in enzymes. Curr Opin Struct Biol. 2004; 14:706-15. [PubMed: 15582395]

3. Jaffe EK. Morpheeins - a new structural paradigm for allosteric regulation. Trends Biochem Sci. 2005; 30:490-7. [PubMed: 16023348]

4. Koshland DE Jr, Nemethy G, Filmer D. Comparison of experimental binding data and theoretical models in proteins containing subunits. Biochemistry. 1966; 5:365-85. [PubMed: 5938952] 
5. Monod J, Wyman J, Changeux JP. On the nature of allosteric transitions: a plausible model. J Mol Biol. 1965; 12:88-118. [PubMed: 14343300]

6. Reitz AB, Ramirez UD, Stith L, Du Y, Smith GR, Jaffe EK. Pseudomonas aeruginosa porphobilinogen synthase assembly state regulators: hit discovery and initial SAR studies. ARKIVOC. 2010 in press.

7. Shanmugam D, Wu B, Ramirez UD, Jaffe EK, Roos DS. Plastid associated porphobilinogen synthase from Toxoplasma gondii: kinetic and structural properties validate therapeutic potential. J Biol Chem. 2010 May 6. [Epub ahead of print].

8. Lawrence SH, Ramirez UD, Selwood T, Stith L, Jaffe EK. Allosteric inhibition of human porphobilinogen synthase. J Biol Chem. 2009; 284:35807-17. [PubMed: 19812033]

9. Lawrence SH, Ramirez UD, Tang L, Fazliyez F, Kundrat L, Markham GD, Jaffe EK. Shape shifting leads to small-molecule allosteric drug discovery. Chem Biol. 2008; 15:586-96. [PubMed: 18559269]

10. Jaffe EK. The porphobilinogen synthase catalyzed reaction mechanism. Bioorg Chem. 2004; 32:316-25. [PubMed: 15381398]

11. Breinig S, Kervinen J, Stith L, Wasson AS, Fairman R, Wlodawer A, Zdanov A, Jaffe EK. Control of tetrapyrrole biosynthesis by alternate quaternary forms of porphobilinogen synthase. Nat Struct Biol. 2003; 10:757-63. [PubMed: 12897770]

12. Selwood T, Tang L, Lawrence SH, Anokhina Y, Jaffe EK. Kinetics and thermodynamics of the interchange of the morpheein forms of human porphobilinogen synthase. Biochemistry. 2008; 47:3245-57. [PubMed: 18271513]

13. Lawrence SH, Jaffe EK. Expanding the concepts in protein structure-function relationships and enzyme kinetics: Teaching using morpheeins. Biochemistry and Molecular Biology Education. 2008; 36:274-283. [PubMed: 19578473]

14. Kokona B, Rigotti DJ, Wasson AS, Lawrence SH, Jaffe EK, Fairman R. Probing the oligomeric assemblies of pea porphobilinogen synthase by analytical ultracentrifugation. Biochemistry. 2008; 47:10649-56. [PubMed: 18795796]

15. Jaffe EK, Stith L. ALAD-porphyria is a conformational disease. Am J Hum Genet. 2007; 80:32937. [PubMed: 17236137]

16. Tang L, Breinig S, Stith L, Mischel A, Tannir J, Kokona B, Fairman R, Jaffe EK. Single amino acid mutations alter the distribution of human porphobilinogen synthase quaternary structure isoforms (morpheeins). J Biol Chem. 2006; 281:6682-90. [PubMed: 16377642]

17. Tang L, Stith L, Jaffe EK. Substrate-induced interconversion of protein quaternary structure isoforms. J Biol Chem. 2005; 280:15786-93. [PubMed: 15710608]

18. Mitchell LW, Jaffe EK. Porphobilinogen synthase from Escherichia coli is a $\mathrm{Zn}(\mathrm{II})$ metalloenzyme stimulated by Mg(II). Arch Biochem Biophys. 1993; 300:169-77. [PubMed: 8424649]

19. Kervinen J, Jaffe EK, Stauffer F, Neier R, Wlodawer A, Zdanov A. Mechanistic basis for suicide inactivation of porphobilinogen synthase by 4,7-dioxosebacic acid, an inhibitor that shows dramatic species selectivity. Biochemistry. 2001; 40:8227-36. [PubMed: 11444968]

20. Walker DA. Regulatory mechanisms in photosynthetic carbon metabolism. Curr Top Cell Regul. 1976; 11:203-41. [PubMed: 11968]

21. Jaffe EK. An unusual phylogenetic variation in the metal binding sites of phorphobilinogen synthase. Chem Biol. 2003; 10:25-34. [PubMed: 12573695]

22. Maruno M, Furuyama K, Akagi R, Horie Y, Meguro K, Garbaczewski L, Chiorazzi N, Doss MO, Hassoun A, Mercelis R, Verstraeten L, Harper P, Floderus Y, Thunell S, Sassa S. Highly heterogeneous nature of delta-aminolevulinate dehydratase (ALAD) deficiencies in ALAD porphyria. Blood. 2001; 97:2972-8. [PubMed: 11342419]

23. Carrell RW, Lomas DA. Conformational disease. Lancet. 1997; 350:134-8. [PubMed: 9228977]

24. Ramirez, UD.; Myachina, F.; Stith, L.; Jaffe, EK. Advances in Computational Biology. In: Hamid, Arabnia, editor. Series: Advances in Experimental Medicine and Biology; Proceedings of the 2009 International Conference on Bioinformatics and Computational Biology; Springer; 2010. scheduled for publication July 2010

25. Li N, Chu X, Liu X, Li D. Probing the active site of rat porphobilinogen synthase using newly developed inhibitors. Bioorg Chem. 2009; 37:33-40. [PubMed: 19095280] 
26. Gacond S, Frere F, Nentwich M, Faurite JP, Frankenberg-Dinkel N, Neier R. Synthesis of bisubstrate inhibitors of porphobilinogen synthase from Pseudomonas aeruginosa. Chem Biodivers. 2007; 4:189-202. [PubMed: 17311232]

27. Frere F, Nentwich M, Gacond S, Heinz DW, Neier R, Frankenberg-Dinkel N. Probing the active site of Pseudomonas aeruginosa porphobilinogen synthase using newly developed inhibitors. Biochemistry. 2006; 45:8243-53. [PubMed: 16819823]

28. Jaffe EK, Kervinen J, Martins J, Stauffer F, Neier R, Wlodawer A, Zdanov A. Species-specific inhibition of porphobilinogen synthase by 4-oxosebacic acid. J Biol Chem. 2002; 277:19792-9. [PubMed: 11909869]

29. Jarret C, Stauffer F, Henz ME, Marty M, Luond RM, Bobalova J, Schurmann P, Neier R. Inhibition of Escherichia coli porphobilinogen synthase using analogs of postulated intermediates. Chem Biol. 2000; 7:185-96. [PubMed: 10712932]

30. Cheung KM, Spencer P, Timko MP, Shoolingin-Jordan PM. Characterization of a recombinant pea 5-aminolevulinic acid dedhdratase and comparative inhibition studies with the Escherichia coli dehydratase. Biochemistry. 1997; 36:1148-56. [PubMed: 9033406]

31. Stauffer F, Zizzari E, Engeloch-Jarret C, Faurite JP, Bobalova J, Neier R. Inhibition studies of porphobilinogen synthase from Escherichia coli differentiating between the two recognition sites. Chembiochem. 2001; 2:343-54. [PubMed: 11828463]

32. Halgren TA, Murphy RB, Friesner RA, Beard HS, Frye LL, Pollard WT, Banks JL. Glide: a new approach for rapid, accurate docking and scoring. 2. Enrichment factors in database screening. J Med Chem. 2004; 47:1750-9. [PubMed: 15027866]

33. Friesner RA, Banks JL, Murphy RB, Halgren TA, Klicic JJ, Mainz DT, Repasky MP, Knoll EH, Shelley M, Perry JK, Shaw DE, Francis P, Shenkin PS. Glide: a new approach for rapid, accurate docking and scoring. 1. Method and assessment of docking accuracy. J Med Chem. 2004; 47:1739-49. [PubMed: 15027865]

34. He MM, Smith AS, Oslob JD, Flanagan WM, Braisted AC, Whitty A, Cancilla MT, Wang J, Lugovskoy AA, Yoburn JC, Fung AD, Farrington G, Eldredge JK, Day ES, Cruz LA, Cachero TG, Miller SK, Friedman JE, Choong IC, Cunningham BC. Small molecule inhibition of TNFalpha. Science. 2005; 310:1022-5. [PubMed: 16284179]

35. Armon-Omer A, Levin A, Hayouka Z, Butz K, Hoppe-Seyler F, Loya S, Hizi A, Friedler A, Loyter A. Correlation between shiftide activity and HIV-1 integrase inhibition by a peptide selected from a combinatorial library. J Mol Biol. 2008; 376:971-82. [PubMed: 18201721]

36. Hayouka Z, Rosenbluh J, Levin A, Maes M, Loyter A, Friedler A. Peptides derived from HIV-1 Rev inhibit HIV-1 integrase in a shiftide mechanism. Biopolymers. 2008; 90:481-7. [PubMed: 18219678]

37. Schechter NM, Choi EJ, Selwood T, McCaslin DR. Characterization of three distinct catalytic forms of human tryptase-beta: their interrelationships and relevance. Biochemistry. 2007; 46:961529.

38. Zhao K, Chai X, Johnston K, Clements A, Marmorstein R. Crystal structure of the mouse p53 core DNA-binding domain at $2.7 \AA$ Åresolution. J Biol Chem. 2001; 276:12120-7. [PubMed: 11152481] 


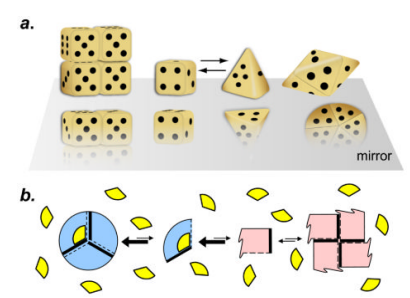

Figure 1. The quaternary structure dynamic characteristic of morpheeins

a) A three dimensional dice analogy is used to illustrate how a die that can morph between a cube and a tetrahedron leads to the assembly of a tetramer and a pentamer respectively. In this case, the common rule of subunit assembly is that "four dots" associate with "one dot". b) The morpheein model of allosteric regulation is illustrated using a two dimensional geometric analogy. A subunit whose conformation is that of a pie wedge (blue) is in equilibrium with a rectangular conformation (pink). The common rule of subunit assembly is that a "dashed line" associates with a "thick line" such that the pie wedge assembles into a circular trimer and the rectangle assembles into a square tetramer. An allosteric regulator, illustrated as a yellow wedge, is shaped to bind preferentially to elements of the trimer. Regulator binding stablizes the blue components of the equilibrium and draws the equilibrium in the direction of the trimer. 


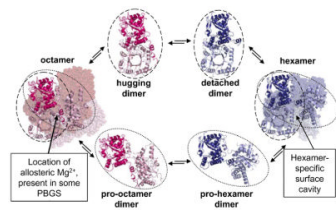

Figure 2. PBGS structure and quaternary structure equilibrium

Each PBGS monomer consists of an $\alpha_{8} \beta_{8}$-barrel domain, the center of which holds the enzyme active site, and an N-terminal arm domain. Some PBGS also contain N-terminal and C-terminal extensions, not shown here. The top pathway (left to right) shows octamer dissociating to hugging dimers, a conformational change to detached dimers, and assembly to the hexamer. The hugging dimer is the asymmetric unit of many PBGS crystal structures; the N-terminal arm of one subunit "hugs" the $\alpha_{8} \beta_{8}$-barrel of the neighboring subunit. The detached dimer, which does not have the hugging interaction, is the asymmetric unit of the crystal structure of the hexameric human PBGS variant, F12L [11]. The location of one hugging and one detached dimer in the octamer and hexamer respectively are shown using dashed ovals. The bottom pathway shows octamer dissociating to pro-octamer dimers, a conformational change to pro-hexamer dimers, and assembly to the hexamer. The positions of one pro-octamer and one pro-hexamer dimer in the octamer and hexamer respectively are shown using dotted ovals. Conformations that support octamer assembly are shown in shades of red; conformations that support hexamer assembly are shown in shades of blue. Light and dark shades are used for contrast in these homo-oligomeric assemblies. 